Heimerziehung o. ä.) nicht erfassen (es sei denn, die entsprechenden Jugendlichen befinden sich in einer „normalen“ JVA), hat das Committee on Crime Problems des Europarats im Januar 2006 eine weitere Expertenkommission beauftragt, Mindestgrundsätze für inhaftierte Jugendliche zu erarbeiten.

16 Vgl. zur Initiative einiger Bundesländer, „Sicherheit" zum gleichwertigen Vollzugsziel neben der Resozialisierung erheben kritisch, Dünkel in NK 2003, S. 8 f. und in Kriminalpädagogische Praxis Heft 43/2004, S. 16 ff.

\section{Konsequenzen der Förderalismusreform für den Deutschen Strafvollzug}

\section{Auszug aus einem Vortrag auf dem 14. Bundeskongress der Psychologin- nen und Psychologen im Strafvollzug am 19. September 2006, Rostock,}

\section{Bernd Maelicke}

W er zahlt, bestimmt" - dies war die Hauptmaxime derjenigen Länder, welche sich vehement für die Zuständigkeitsverlagerung vom Bund auf die Länder eingesetzt hatten. 10 Länder arbeiten bereits an einem inhaltlich übereinstimmenden Entwurf für die bis zum Jahresende 2007 zu verabschiedenden Landes-Jugendstrafvollzugsgesetze ( so die Vorgabe des Bundesverfassungsgerichts, besprochen in diesem Heft von Dünkel (Rechtsprechung) und Ostendorf (Titel). Bisher liegen nur von Bayern und Baden-Württemberg erste Diskussionsentwürfe ohne Begründung und ohne Abstimmung mit den zuständigen Stellen vor - sie haben noch einen langen parlamentarischen Weg vor sich, sodass sie $z . Z t$. noch nicht bewertet werden
senen-Vollzug geben wird. Eckpunkte liegen z.B. aus Niedersachsen vor - aber noch keine veröffentlichten Entwürfe. Brennpunkte der Diskussion in den Landtagen werden z.B. sein:

- Gewichtung der Vollzugsziele Behandlung der Gefangenen und Schutz der Allgemeinheit

- Umfang und Qualität der Behandlungsangebote (insbes. Arbeit der Gefangenen)

- Ausgestaltung der Lebensverhältnisse im Vollzug (z.B. Wohngruppen, Haftraum-Ausstattung, Besuchs- und Telefonregelungen, Kostenbeteiligungen)

- Einzel-/Gemeinschaftsunterbringung

- Inhalt und Umfang der Vollzugslockerungen

- Urlaubsregelungen

- Entlassungsvorbereitung, Übergangsmanagement, Nachsorge

- Personalausstattung

- Innerer Aufbau und Organisation der Anstalten

- Teilprivatisierung

- Wirkungsorientierte Steuerung (Controlling).

Die Finanzpolitiker werden viele Versprechungen und Wunschträume von Erhöhungen der Standards zunichte machen - zu katastrophal ist die Finanzlage der Länder - zu hoch der unabweisbare Finanzbedarf im Elementarbereich, in den Schulen, in der Jugendhilfe, in der Altenhilfe, in den Universitäten, bei der Polizei. Die Gefahr der Absenkung des Niveaus ist mehr als realistisch - die Abgeordneten haben klare Vorstellungen darüber, welche Prioritäten ihre Wähler setzen. Bei jedem Sicherheitsvorfall wird der Landesgesetzgeber gefordert sein - hier wird ein Wettbewerb über höchstmögliche Standards zu erwarten sein - koste es, was es wolle! Schon bei den Regelungen auf Bundesebene wurde ein gesetzgeberisches Gesamtkonzept für die ambulante und stationäre Resozialisierung angemahnt - dies gilt nun erst Recht für die Länder, die auch für die Gerichtshilfe, die Bewährungshilfe, die Führungsaufsicht und die Freie Straffälligenhilfe die Zuständigkeit haben. Es stellen sich fachlich und organisatorisch anspruchsvolle und aufwändige Innovationsnotwendigkeiten - noch ist nicht sichtbar, dass sich insoweit ein Land (entsprechend internationalen Vorbildern) profilieren will. Der gesetzgeberische und bürokratische Aufwand in den Ländern verursacht schon jetzt erhebliche zusätzliche Kosten, da förmliche Landesgesetze (Strafvollzug, Jugendstrafvollzug, U-Haft), Verwaltungsvorschriften (Neufassung der bisherigen bundeseinheitlichen Verwaltungsvorschriften und der wesentlich zahlreicheren ergänzenden Verwaltungsvorschriften der Länder) und Erlasse (Anpassung aller bisherigen Erlasse an die neuen Regelungen) nötig werden. Vernachlässigt und übersehen wurde bisher das Thema Jugendarrest auch hier fehlt es an den erforderlichen gesetzlichen Grundlagen für diese gravierende Form der Freiheitsentziehung - das Bundesverfassungsgericht ist aufgerufen zur gesetzlichen Regelung der Untersuchungshaft und des Jugendarrests klare Fristen zu setzen.

Fachfragen dürfen nicht zu Machtfragen werden. Eine kontinuierliche und professionelle länderübergreifende und europaweit als Vorbild dienende Systementwicklung und Qualitätssteigerung ist abrupt unterbrochen worden. Nun ist die Vollzugspraxis aufgefordert, zusammen mit den Fachministerien, der Wissenschaft, den Fachorganisationen auf Länderebene das Beste aus dieser Situation zu machen. Es müssen neue regionale und länderübergreifende Netzwerke und Unterstützungssysteme aufgebaut/verstärkt werden. Die Länder-Rechtsprechung wird größere Bedeutung bekommen, die Kommentatoren werden an Gewicht verlieren. Der Energieverschleiß ( Personal- und Sachkosten ) wird immens sein - diese Potentiale werden vor Ort bei der Gewährleistung von guter Behandlung und optimierter Sicherheit zumindest in absehbarer Zeit fehlen.

Bernd Maelicke war seit dem 1. Heft vor mittlerweile 20 Jahren Mitherausgeber dieser Zeitschrift. Wir danken ihm für sein Engagement. Er wird nun die Zeitschrift für Strafvollzug und Straffälligenhilfe als Schriftleiter übernehmen, da der geschätzte Kollege Heinz Müller-Dietz aus Altersgründen diese Aufgabe abgibt. 